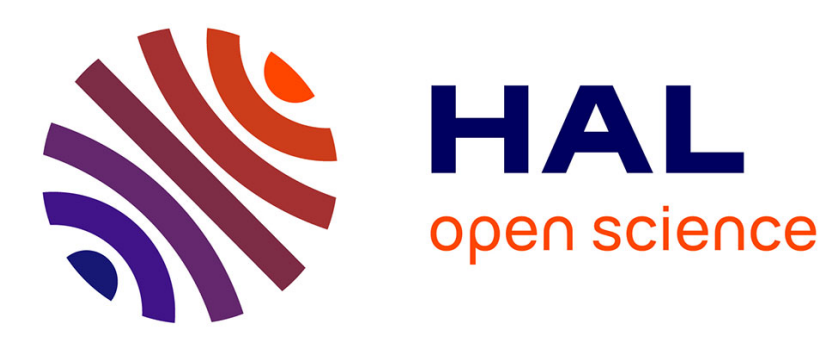

\title{
Nonequilibrium biochemical structures in two space dimensions with local activation and regulation
}

\author{
Bertrand Fourcade
}

\section{To cite this version:}

Bertrand Fourcade. Nonequilibrium biochemical structures in two space dimensions with local activation and regulation. Physical Review E , 2020, 101 (1), 10.1103/PhysRevE.101.012420 . hal02465425

\section{HAL Id: hal-02465425 \\ https://hal.science/hal-02465425}

Submitted on 3 Feb 2020

HAL is a multi-disciplinary open access archive for the deposit and dissemination of scientific research documents, whether they are published or not. The documents may come from teaching and research institutions in France or abroad, or from public or private research centers.
L'archive ouverte pluridisciplinaire HAL, est destinée au dépôt et à la diffusion de documents scientifiques de niveau recherche, publiés ou non, émanant des établissements d'enseignement et de recherche français ou étrangers, des laboratoires publics ou privés. 


\title{
Nonequilibrium biochemical structures in two space dimensions with local activation and regulation
}

\author{
B. Fourcade \\ Grenoble-Alpes University, CNRS, LIPHy, 38000, Grenoble, France.
}

(Dated: January 7, 2020)

\begin{abstract}
Integrin receptor (IR) clustering is an example of pattern self-organization in biological systems. We study a model for receptor activation whose content is guided by two major principles in cellular signal transduction: (i) Proteins cycle between different conformational states; (ii) The dynamics of their conformational dynamics is environment dependent. Based on a simple activation pathway where these two hypotheses are formulated in a self-consistent way, this paper focuses mainly on stochastic simulations valid in the limit of a small number of molecules. It is shown that coherent clustering can lead to digital signaling and receptor competition in biochemical systems where the model gives a recruitment mechanism for the reinforcement of the mechanical linkage with the extracellular matrix. Together with previous works, this paper provides a workable model for cell integrin adhesive structures when feedback mediated by membrane diffusing signals is dominant. Consequences are discussed in the framework of published data concerning the local production of a key phospholipid for cell signaling $\left(\mathrm{PIP}_{2}\right)$.

PACS numbers: 87.17Rt,87.17Aa,8716Xa,05.65+b
\end{abstract}

\section{INTRODUCTION}

One of the most striking and ubiquitous phenomena in biology is the emergence of self-induced and self-sustained structures. These spatial and temporal structures are archetypes of pattern formation whose self-organization principles are only partly understood[1-5]. In particular, adhesive complexes for cells on substrate grow from nascent adhesions formed of integrin receptors (IR) with no apparent elasticity and develop into more rigid structures connected to the cytoskeleton. Other adhesive structures, called podosomes[6], are characteristic of invasive cells and form a dynamic bestiary of shapes including mesoscopic rings. One ubiquitous property of these structures is the recruitment of IRs into high-density organized clusters to serve as a hub and to process information[7]. The formation of IR-rich clusters having crucial effects on biological responses, it is relevant to study generic mechanisms for their formation.

To study the early stage of IR clustering, the present paper concentrates on a feedback mechanism with a membrane diffusive signal. Since nascent adhesions use adhesome components independently of mechanical forces[8], we focus in this paper on signaling properties for receptor clustering using a reaction network where pattern formation is only due to reaction-diffusion processes. Models for clustering such as the purely mechanical trapping of ref. [9, 10] or other general models for signaling components, including lipid rafts and cytoskeleton linkages, have pointed out the role of feedback in spatial clustering of molecules for efficient digital signaling[11] and cell polarization mechanisms [12]. In these works, positive feedback is generally associated with a molecule promoting its own production and it has been shown that this mechanism leads nanoclusters of active molecules[13, 14].

Changes in membrane composition play, however, a key role in IR clustering[15]. It is therefore natural to study how a diffusive membrane signal triggers the emergence of selfsustained IR structures with size comparable to its diffusion length when this signal is locally produced at adhesive sites.
In this paper, such a diffusive signal plays the role of an activator and the model mimics key properties of phosphoinositide signaling on IR regulation. In particular, phosphoinositides such as $\operatorname{PtdIns}(4,5) \mathrm{P}_{2}$, i.e. $\mathrm{PIP}_{2}$, are known to regulate actin polymerization factors $[16,17]$ and the membrane cytosol interface by recruiting signaling components $[15,18]$ which, in turn, activate IRs[19-21]. In particular, superresolutive microscopies[22, 23] have shown that a large fraction of IRs are mobile within adhesive clusters with different types of IRs exhibiting distinct dynamics and different spatial organizations $[24,25]$ in a constantly redistributed environment[25] .

In the classical continuum framework for a diffusionreaction model, Ref.[26] has already shown that local production of an activator is enough to cluster IRs. This raises the question whether this model is still valid and what are the different phases in a regime where the number of molecules is small and where the intrinsic noise due to diffusion and reaction dominates.

To this end, we introduce a more general molecular pathway model appropriate to stochastic simulations which can be generalized to model more mature adhesive structures. As before, the model is guided by two principles in signal transduction: IRs cycle between active and inactive state and the rate of this cycling depends on membrane composition which is determined in a self-consistent way. This model reproduces the sequence of phases (homogeneous, localized spikes, expanding incomplete or complete rings) for different excitation levels. The main difference between the stochastic and the deterministic approach is the absence of the self-replicating spot instability with the presence of expanding incomplete arcs in a circle reminiscent of podosome mesostructures. The use of stochastic framework is also appropriate when local production of the activator induces crosstalks and phase segregation for a mixture of receptors competing for immobilization sites. In particular, this paper establishes a casual link between the dynamics of receptors and how one type of receptor can regulate the activation state of a different one[27, 28]. As a result, the IRs with the highest residence time are favored and this 
differential recruitment mechanism predicts a reinforcement for the force transmission through adhesive structures as in other models which rely on a signal to increase IR concentration, see $[29,30]$ and refs. therein.

The biological significance of theses results is discussed in the conclusion where this work is put into its biological context with a summary of published data concerning $\mathrm{PIP}_{2}$ signaling and IR clustering. To facilitate the reading, analytical results concerning the conditions under which selfsustained structures exist are reported in Appendix. Movies from the stochastic simulations are provided as supplementary material[31].

\section{CONTINUUM MODEL FOR RECEPTOR CLUSTERING}

Following [26], the model is divided into 3 elementary modules, see Fig. 1 together with Refs. [15, 18-20] for their biological significance. The first is a change in membrane composition where $u(\mathbf{x}, t)$ serves as a switch for IR activation. This module simulates local changes in lipid concentration $\left(\mathrm{PIP}_{2}\right)$ to recruit cytosolic IR activators such as Talin. For this reason, $u(x, t)$ is an activator. Second, IRs are represented with a two-state density field, $\left(n_{i}(\mathbf{x}, t), n_{a}(\mathbf{x}, t)\right)$. Receptors switch between their inactive and active states with rates $\omega_{ \pm}(u)$, and an increase in $u$ makes the active state stable. Inactive receptors are free to diffuse, but active ones are immobile, because of their strong affinity to external ligands. In cells, the kinetics of $\mathrm{PIP}_{2}$ synthesis and hydrolysis determines when and where a $\mathrm{PIP}_{2}$ regulated protein can be activated and local production of PIP $_{2}$ by the lipid kinase PIPKi $\gamma$ serves to recruit new proteins. For this reason, a third field $v(\mathbf{x}, t)$ is introduced for the local production of $u(\mathbf{x}, t)$. This rate of production of $u(\mathbf{x}, t)$ is proportional to the product of the local density of receptors by $v(\mathbf{x}, t)$, i.e. $v(\mathbf{x}, t) n_{a}(u)$. Since the depletion of $v(\mathbf{x}, t)$ decreases the production of $u(\mathbf{x}, t)$, this field is a regulator.

In the continuum limit, the pair $(u, v)$ obeys the general equations of motion :

$$
\begin{aligned}
& \partial_{t} u=d_{u} \Delta u+\frac{1}{\varepsilon}\left[-b u+v n_{a}(u)\right] \\
& \partial_{t} v=d_{v} \Delta v+h\left(v_{c}-v\right)-f(v) n_{a}(u)
\end{aligned}
$$

where $d_{u}, d_{v}$ are diffusion constants and $b / \varepsilon$ is a rate constant for the irreversible degradation of $u$. The last term in the second equation, $f(v) n_{a}(u)$, couples the dynamics of $v$ to the concentration $u$ and $h\left(v_{c}-v\right)$ simulates the exchange of $v$ between the membrane and the cytosol. The function $f(v)$ is general. Since a cluster of receptors indicates a high density of $u$, and thus a small density of $v$, we can Taylor expand $f(v)$ for small $v$ as $f(v)=a_{0}+a_{1} v+\ldots, a_{1} \geq 0$.

To complete the system, the equation of motion for $\left(n_{i}, n_{a}\right)$ follows from the kinetics

$$
\begin{aligned}
\partial_{t} n_{i} & =d_{n} \Delta n_{i}-\omega_{+}(u) n_{i}+\omega_{-}(u) n_{a} \\
\partial_{t} n_{a} & =+\omega_{+}(u) n_{i}-\omega_{-}(u) n_{a}
\end{aligned}
$$

where $d_{n}$ is the diffusion constant of inactive IRs. Finally, the coupling between the kinetic rates $\omega_{ \pm}(u)$ and the environment

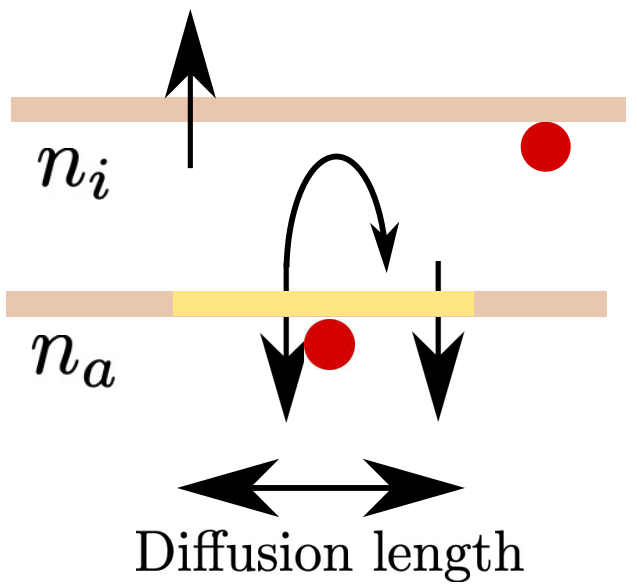

FIG. 1. Schematic representation of the three modules studied in this paper. Arrows correspond to IRs in their inactive/active-immobile states $\left(n_{i}, n_{a}\right)$. A change in color in the bar code indicates a local change in composition $u$ within the membrane corresponding to lipid production, i.e. $\mathrm{PIP}_{2}$. An increase of $u$ makes active IRs more stable and, for this reason, $u$ is an activator. The loop arrow represents the local production of the activator $u$ which serves as a positive feedback loop to recruit new receptors in their active state. Regulation of lipid production is controlled by a third field $v$ (red dots) when IRs are immobile. This field $v$ mimics the recruitment of specific kinases such as PIPKI $\gamma$ to adhesive sites.

$u(x, t)$ is introduced if $u(x, t)$ is interpreted as a chemical potential with

$$
\omega_{-}(u) / \omega_{+}(u)=\omega_{-}^{0} / \omega_{+}^{0} e^{-\beta u}
$$

where $\beta$ is a positive constant and $\omega_{-}^{0} / \omega_{+}^{0} \gg 1$ so that the inactive state is favorable in the low $u$ limit and switches to the active state in the other limit. This sets a lower bound for the characteristic excitation in the spike as

$$
u_{c}=\frac{1}{\beta} \ln \frac{\omega_{-}^{0}}{\omega_{+}^{0}}
$$

In the model considered here, however, all active IRs are immobilized to external ligands, and $1 / \omega_{-}(u)$ should be interpreted as a residence time which is typically much longer than a conformational interconversion rates[32]. The chemical potential difference between the two conformations should also depend logarithmically on the cytosolic concentration of an activator protein, but this log-dependence is subdominant with respect to the linear dependence in $u$. For simplicity, this term will be neglected. However, since $u$ serves as docking sites for large proteins such as Talin which regulate IR activation, the effective diffusion of $u$ is affected and is less than for a usual lipid. This work assumes, therefore, that components have almost equal diffusion constants, $d_{u} \simeq d_{v} \simeq d_{n}$.

As shown in the inset of Fig. 2, the problem defined by (1) together with (2) has a unique fixed point $\left(u_{h}, v_{h}\right)$ at low $u$ for a broad range of parameters. Neglecting diffusion, the shape of typical trajectories in the $(u, v)$ plane demonstrates that this system is excitable because of the following reason. All initial conditions within a small neighborhood of a stable 


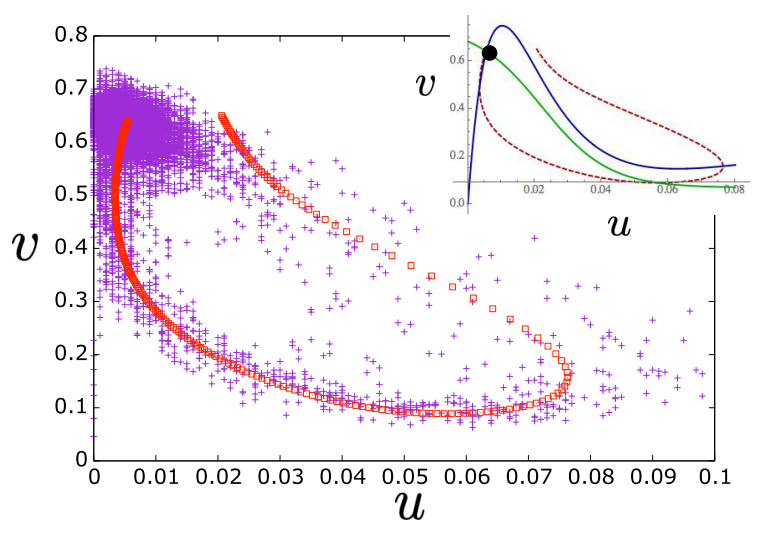

FIG. 2. Nullclines and trajectories $(v(t), u(t))$ for problem (1) in the limit of constant receptor density. The inset shows a typical trajectory when the system is perturbed away of its homogeneous fixed point $\left(u_{h}, v_{h}\right)$ where the two blue and red curves intersect. The main figure represents the same time trace trajectory for the equivalent problem but in the stochastic limit with the emergence of a stochastic limit cycle absent in the deterministic case. This limit is studied in section III with a reference receptor density per compartment of 1000 .

fixed point give trajectories that decay directly to it, but some initial conditions further away from the stable fixed point give trajectories that undergo large excursions before approaching the fixed point. These excursions are controlled by the parameter $\varepsilon$ in (1), so that when $\varepsilon$ is small, large excursions are expected.

Following a delta-like initial perturbation, numerical integration of the time-dependent equations of motion (1) + (2) converges to localized static or dynamic self-sustained structures as in Fig. 3. These structures exist only in some regions of the parameter space where the system is excitable enough[26] and a typical phase diagram is shown in Fig. 4. As studied in Appendix A, these structures are localized due to local production of the activator $u$ with clusters of active IRs on a scale comparable to the diffusion length $l_{u}=\sqrt{d_{u} \varepsilon / b}$. The other length scale characterizing the problem is set by the diffusion length of the activator, $l_{v}=\sqrt{d_{v} / \tilde{h}}$, where $\tilde{h}$ differs little from $h$. As shown in Appendix A, the characteristic scale for the activator in the spike is set by the ratio $d_{v} v_{h} /\left(d_{u} \varepsilon\right)$ with logarithmic corrections. To compensate for the consomption of $v$ within the spike, the recruitment of $v$ by lateral diffusion must be strong enough. This sets a condition on the diffusion constant of the regulator $v$ and comparing with (4) gives $d_{v} / d_{u}>\varepsilon u_{c} / v_{h}$ as a lower bound. Because of these strong logarithmic corrections present even for $l_{v} \approx 10 l_{u}$, this asymptotic criterion gives only trends when compared with numerical data, but this anaysis has the avantage of identifying key relevant parameters.

Static solutions such as the spikes or the rings of Fig. 3 correspond to a regime where diffusion and reaction are uncoupled. Receptors can diffuse rapidly through IR cluster before being immobilized so that IR concentration can be consider-
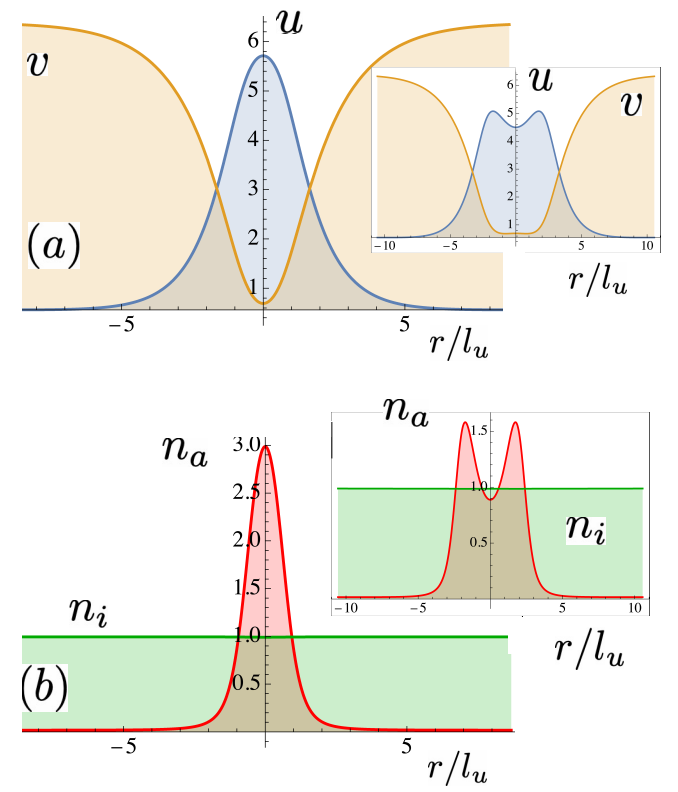

FIG. 3. Plots of stable spike solutions with axial symmetry of rotation. Case (a) corresponds to the activator $u$ in blue and the regulator $v$ in orange. The figure corresponds to a spike solution on the right boundary line of Fig. $4\left(\varepsilon=5.91, d_{v}=d_{u}=1\right)$. The insets show the solution for parameters on the ring boundary line ( $\varepsilon=4, d_{v}=d_{u}=1$ ). Subfigure (b) represents the variations for the inactive (green) and for the active integrin receptors for the same values of the parameters. Note that the density of inactive IRs has converged to one following an initial delta-like perturbation. Going from the spike to the ring solution can be realized by varying the excitability parameter $\varepsilon$ or the kinetic rate for membrane recruitment $h$.

ably increased. This regime imposes, therefore, a maximum size for the cluster and this criterion is consistent with experimental observations that IRs enter and exit focal adhesions with a significant fraction of mobile IRs[22, 33].

\section{STOCHASTIC SIMULATIONS}

\section{A. A molecular model}

For stochastic simulations, it is useful to consider a prototypical molecular pathway of Fig. 5. This biochemical network can be reduced to the model we have considered so far, but this form allows to point out a key irreversible step in the signaling pathway. In order to distinguish with the continuum limit, concentrations are represented with capital letters. As before, we assume that all active IRs are immobile and neglect in a first stage any change in free ligand concentration. 


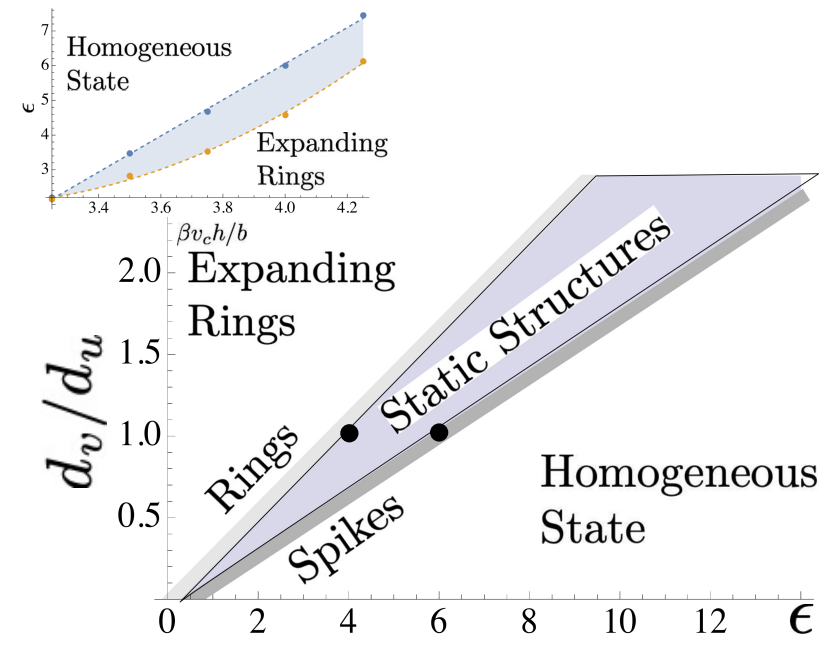

FIG. 4. Schematic phase diagram of the problem (1). The $x$ and $y$ coordinates represent the excitability parameter and the ratio of the diffusion constants for the activator $u$ and the regulator $v$. For a given ratio $h v_{c} / b$, the phase plane is separated into three different regions. At low excitability (large $\varepsilon$ ), on the right part of the figure, the system returns to the quiescent homogeneous state following a delta-like excitation. Crossing the boundary lines gives static excited solutions. The shape of these solutions are either spikes on the righthand side of the gray domain or rings on the left, see Fig. 3. For small ratios $d_{v} / d_{u}$, expanding rings are only transient solutions for other sets of parameters. The inset shows the same phase diagram but in the $\left(h v_{c} / b, \varepsilon\right)$ plane for equal diffusion constants $d_{v} / d_{u}=1$. Increasing the flux $h v_{c}$ makes the system to bifurcate from static to the expanding rings (adapted from Ref.[26], $h=0.1, b=0.2, \beta=$ $\left.1, \omega_{-}^{0} / \omega_{+}^{0}=100\right)$. The two dots correspond to the parameters of Fig. 3 with $\left(u_{h}, v_{h}\right) \approx(0.56,6.4)$.

A protein $\mathrm{V}$ binds an immobilized receptor and undergoes an irreversible post-translational modification which affects its binding to the complex $\mathrm{I}_{a}-\mathrm{V}$. The modified form $\varnothing$ is inactive for the production of $\mathrm{U}$ and loses its anchor site to the membrane. The down-regulation of the active form of $V$ can also be accomplished through competition for binding by other cytoskeletal proteins and we define the dwell time $\tau$ whose typical order of magnitude corresponds to a few phosphorylation-dephosphorylation cycles. During this dwell time, the complex $\mathrm{I}_{a}-\mathrm{V}$ produces $U$ at a rate $g_{\text {prod }}$. In this picture, the depletion of $V$ occurs in tandem with an accumulation of $\mathrm{I}_{a}-\mathrm{V} . V$ must, however, be continuously fed into the system because of the irreversible step

$$
\mathrm{I}_{a}-\mathrm{V} \stackrel{1 / \tau}{\longrightarrow} \mathrm{I}_{a}+\varnothing
$$

The model can easily generalized by introducing a series $\left(\mathrm{I}_{a}-\mathrm{V}\right)_{i}$ of intermediate states before this irreversible step without any changes. As before, the dynamics of $U$ is written with the source term proportional to the concentration $\mathrm{I}_{a}-\mathrm{V}$

$$
\frac{\partial \mathrm{U}}{\partial t}=D_{u} \Delta \mathrm{U}-B U+g_{\text {prod }} \mathrm{I}_{a}-\mathrm{V}
$$

In the compartment based approach taken here, the diffusion constants are appropriately renormalized as discussed later.

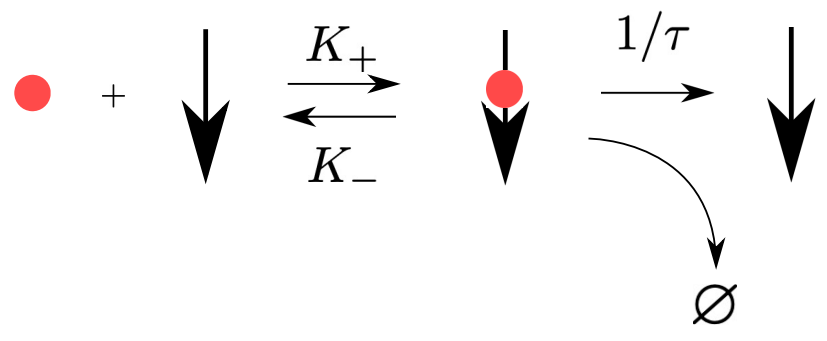

FIG. 5. Schematic pathway considered in the stochastic section. The molecule $\mathrm{V}$ (red dot) binds to an immobilized integrin receptor $I_{a}$ (down arrow) to form a complex $I_{a}-V$. A covalent modification of $V$ alters the affinity of $\mathrm{V}$ to $\mathrm{I}_{a}, \mathrm{~V} \longrightarrow \varnothing$. As a consequence, $\varnothing$ unbinds from $I_{a}$ after a characteristic binding time $\tau$ where it loses its anchor site to the membrane.

Let $K_{ \pm}$be the binding and unbinding rates of $V$ to $I_{a}$. Since $\tau$ is the typical time for a post-translational modification and $1 / K_{-}$is the binding time for a biochemical bond, we expect $1 / \tau \ll K_{-}$. Assuming that the concentration of the complex $\mathrm{I}_{a}-\mathrm{V}$ can be approximated by its pseudo-equilibrium value[34], we have:

$$
\mathrm{I}_{a}-\mathrm{V}=\frac{K_{+}}{K_{-}+1 / \tau} \mathrm{I}_{a} \times \mathrm{V}
$$

so that the equation for the dynamics of $V$ is

$$
\frac{\partial \mathrm{V}}{\partial t}=D_{v} \Delta \mathrm{V}+H\left(\mathrm{~V}_{c}-\mathrm{V}\right)-\frac{1}{\tau} \mathrm{I}_{a}-\mathrm{V}
$$

Eqs. (6) and (8) correspond the deterministic model studied so far. The quasi-equilibrium (7) approximation simplifies the problem but will be relaxed in the numerical simulation.

To proceed, it is convenient to scale the number of molecules in compartment $i, \mathrm{U}_{i}$, by reference with the averaged number of receptors $N_{0}$ per compartment. The number of molecules in a compartment $i$ and the densities are related by

$$
\frac{U}{N_{0}}=\frac{u}{n_{0}}
$$

The deterministic binding rate is thus related to binding rate per compartment as $k_{+}=N_{0} K_{+}$for $d\left[\mathrm{I}_{a}-\mathrm{V}\right] / d t=K_{+} \mathrm{I}_{a} \mathrm{~V}$ to be equivalent to $d\left[n_{a}-v\right] / d t=k_{+} n_{a} v$. Table I summarizes the correspondence between the parameters for the stochastic molecular model with their deterministic PDE counterparts, so that the nullclines of Fig. 2 can be compared in the two cases.

After appropriate rescaling, (8) and (6) are therefore equivalent to problem (1) with an effective dimensionless parameter

$$
\frac{1}{\varepsilon}=g_{\text {prod }} \tau
$$

The value of the molecular model is, therefore, to give a physical meaning of the dimensionless parameter $\varepsilon$ as the ratio of 


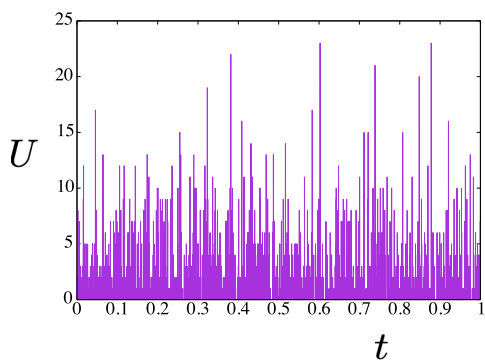

FIG. 6. Single cell stochastic simulation: Number of $U$ molecules as a function of time in the case of a single compartment (homogeneous case). The average number of free integrin receptors per compartment is $N_{0}=10$. This simulation shows that the stochastic dynamics of the activator $u$ exhibits strong irregular bursts above the homogeneous fixed point value $(\langle u\rangle=0.41,\langle v\rangle=3.91)$ given by the deterministic case.

\begin{tabular}{|l|r|}
\hline PDE & Stochastic \\
\hline$k_{+}$ & $N_{0} K_{+}$ \\
$k_{-}$ & $K_{-}$ \\
$1 / \varepsilon$ & $g_{\text {prod }} \tau$ \\
$b / \varepsilon$ & $\left(1+k_{-} \tau\right) / k_{+} B$ \\
$v_{c}$ & $V_{c} / N_{0}$ \\
$h$ & $\left(1+k_{-} \tau\right) / k_{+} H$ \\
\hline
\end{tabular}

TABLE I. Correspondence between the stochastic molecular model and the deterministic framework, see Eqs (1). $K_{ \pm}$are the bindingunbinding rates of the regulator $V$ to the receptor $I$. Typical values corresponding to Fig. 6 are $K_{+}=0.01, K_{-}=1.0, H=0.005, V_{c}=$ $7.5, g_{\text {prod }}=0.25, \beta U=10 U$.

two well-known regulators in cell signaling, i.e. kinase activity and lifetime for a the activation of a molecular architecture.

To complete this reaction scheme, we consider the occupancy probabilities for $N$ discrete compartments of a finite lattice. Let $\left(\mathrm{I}_{i}, \mathrm{I}_{a}, \mathrm{U}, \mathrm{V}, \mathrm{I}_{a}-\mathrm{V}\right)_{i}$ be the number of molecules in compartment $i$. Our method is standard and we follow Refs. $[35,36]$. Each compartment is considered homogenous and compartments are coupled via diffusion. An event is either a chemical reaction taking place in a compartment or a stochastic jump from one compartment to its neighbors. The Gillepsie's algorithm samples the joint probability distribution for occupancy by simulating the distribution rate for each event and choose the next event to take place[35]. A conformational change of a receptor in a compartment $i$ is considered as an event and the rates $\omega_{ \pm}\left(U_{i}\right)$ in compartment $i$ depend on the number of activator molecules $U_{i}$ in the same compartment. As before, active receptors are assumed to be bound to a ligand and are immobile.

Diffusion is described by the following chain of reactions between nearest neighbor compartments for any specie $\mathrm{X}_{i}$ :

$$
\mathrm{X}_{1} \underset{\mathrm{DX}_{\mathrm{X}}}{\stackrel{\mathrm{D}_{\mathrm{X}}}{\rightleftharpoons}} \mathrm{X}_{2} \underset{\mathrm{D}-\mathrm{X}}{\stackrel{\mathrm{DX}_{\mathrm{X}}}{\rightleftharpoons}} \mathrm{X}_{3} \underset{\mathrm{DX}_{\mathrm{X}}}{\stackrel{\mathrm{D}_{\mathrm{X}}}{\rightleftharpoons}} \ldots \underset{\mathrm{DX}_{\mathrm{X}}}{\stackrel{\mathrm{D}_{\mathrm{X}}}{\rightleftharpoons}} \mathrm{X}_{\mathrm{N}}
$$

The effective diffusion constant $D_{X}$ is scaled by the cell size $l$

$$
D_{X}=d_{x} / l^{2}
$$

where $d_{x}$ is the usual diffusion constant when the model is written with surface fraction $x$ as in (1). The definition of the size $l$ is arbitrary. It is convenient to define $l$ as approximately half the size of the diffusion length of $u$ which is the smallest length scale entering in the problem

$$
l=1 / 2 l_{u}=\frac{1}{2} \sqrt{d_{u} \varepsilon / b}
$$

so that $D_{u}$ can be directly computed and all other diffusion constants appropriately renormalized. For all the results detailed below, the diffusion constants of the different species are comparable and we use a single compartment size to avoid undesirable bias[36, 37]. Using the correspondences of table I, Fig. 2 compares the phase space trajectories of problem (1) in both its stochastic and deterministic versions. Since the stochastic version does not assume stationary equilibrium for the complex $\mathrm{I}_{a}-\mathrm{V}$, Fig. 2 is the projection on the $u-v$ plane of the actual trajectory. Although the traces in two figures appear analogous, the smallness of the number of particles triggers a self-induced stochastic resonance with the apparition of a stochastic limit cycle absent without noise[38]. The appearance of this limit cycle obtained for $N_{0} \sim 1000$ molecules is, however, considerably degraded for a smaller number, e.g $\sim 10$, where the time series of $u(t)$ exhibits strong bursts emerging from a very noisy signal presenting irregular sequences, see Fig. 6.

As a last point, we discuss how appropriate boundary conditions are implemented with solutions matching their homogeneous fixed point value $U_{h}, V_{h}, N_{0}$ at infinity. For this, we simulate a probabilistic thermostat in contact with the boundaries. Since molecules outside the simulation box are assumed to diffuse freely, the joint probability to find $U_{i}, V_{i}$ or $I_{i}$ particles in compartment $i$ along the boundaries is a multinomial distribution given by their mean values $U_{h}, V_{h}$ or $N_{0}$. From a practical point of view, a periodic update of the compartments along the edges by drawing the number of particles according to this distribution simulates this probabilistic Andersen thermostat. Systems from $50 \times 50$ to $100 \times 100$ compartments can be simulated at a reasonable computing time.

\section{B. Results in the stochastic case}

The main results of this section are summarized by the density plots of Fig. 7 for different runs along a line parallel to the $\varepsilon$-axis of Fig. 4 with their corresponding movies in the supplementary data. When diffusion is taken into account, stochastic fluctuations are not able to excite the system enough and the homogenous state is characterized by bursts of active receptors with transient small clusters. Receptor clustering is only induced by a threshold perturbation. Following a delta like excitation with a few tenths of $\mathrm{I}_{a}-\mathrm{V}$ molecules in the compartment at the center of simulating domain, one obtains series of snapshots representative of the difference phases. As expected, when the excitability is small, i.e. for $\varepsilon$ sufficiently large, a delta-like excitation slowly fades away and the system returns to the homogenous phase. Receptor clustering characterized by a pattern of high density of active receptors occurs 
(a)
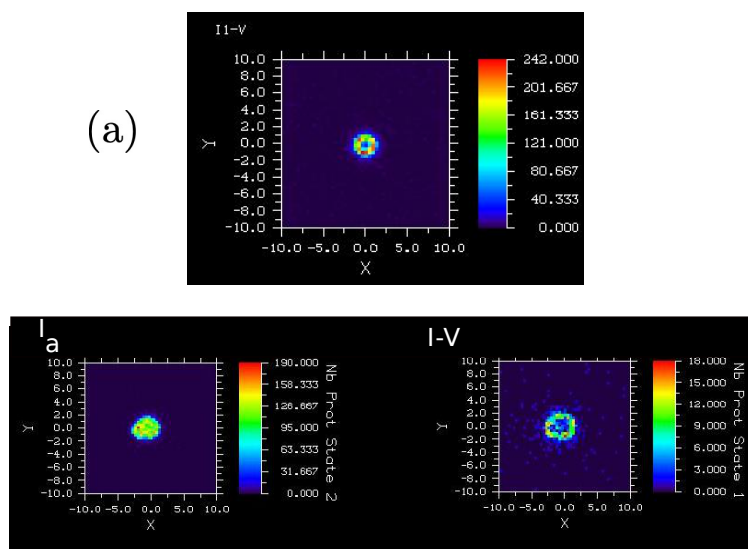

(b)

(c) (d)

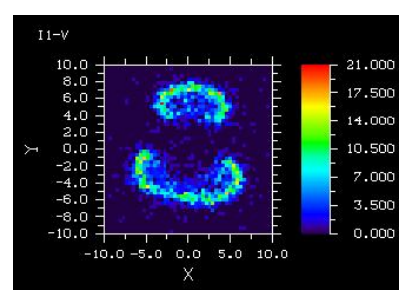

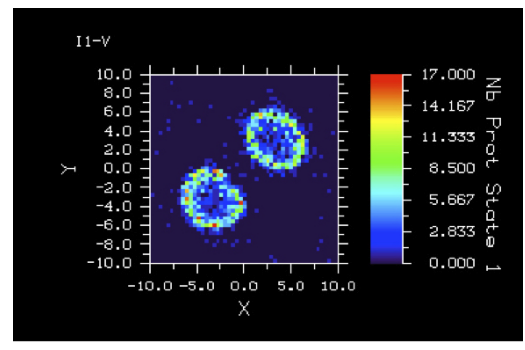

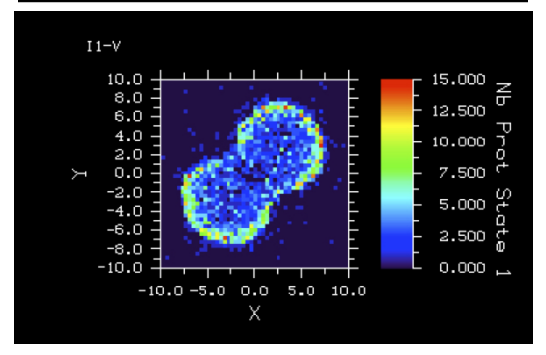

FIG. 8. Expanding rings. Case (a) corresponds to a snapshot for two expanding rings. For this set of parameters, the depletion-mediated interaction between the two rings distorts the circular shape of the rings which move away to optimize the diffusion of $V$. Case (b) corresponds to an annihilation dynamics where the two rings fuse in one (simulating box: $50 \times 50$ ).

FIG. 7. Examples of density plot for stochastic self-sustained structures taken from snapshots of numerical calculations. Case (a) represents a static spike with a reference number of IRs $N_{0}$ equal 100. Immobile-active receptors $\mathrm{I}_{a}$ and complexes $\mathrm{I}-\mathrm{V}$ are roughly equally distributed with a slight increase of $\mathrm{I}-\mathrm{V}$ at the rim. For all other cases, $N_{0}=10$. Case (b) is the snapshot of a static spike where the $\mathrm{I}-\mathrm{V}$ molecules form a fluctuating ring at the rim of the spike. The left panel, (b), corresponds to active receptors $\mathrm{I}_{a}$ and the right panel, (c), to the complex I-V. I-V complexes are distributed at the rim of the adhesive spike. Case (d) is the snapshot of an expanding complete ring which becomes incomplete above a critical radius with spiraling ends. Typical parameter values corresponding to (b) are: $\omega_{+}(0)=0.001, \omega_{-}(0)=1, H V_{c}=2, H=0.05, K_{+}=0.1, K_{-}=$ $1, \tau=1, g_{\text {prod }}=3.8, B=0.1425$. For companion movies, see supplemental data.

only at large enough excitability. When the average number $N_{0}$ of receptors is large, i.e. of the order of 100, the static spike of the deterministic framework is reproduced. For lower $N_{0}$, i.e. a few 10 the static spike experiences strong breathing deformations and exhibits an intermittent depletion hole in the middle, see case (a), indicating local segregation between the receptors. Even for this relatively large number of molecules, fluctuations are huge and the dynamics processes via bursts. Case (b) of the same figure shows a static spike where the I-V complexes form a ring at the rim of the spike with active receptors centered in the spike (see SM for movies[31]). This gradient reflects the strong turnover of $\mathrm{V}$ molecules which diffuse from the outside of the spike and leave active integrins $\mathrm{I}_{a}$ in the center of the spike. Complete expanding rings are observed in the large excitation regime. In the medium excitation regime, but still in the dynamic part of the phase diagram, complete expending rings break into circular arcs above a critical radius and form 'croissants' with spiraling ends. The size of this critical radius is controlled by the parameters and can be bigger than the simulating box.

In marked contrast with its deterministic counterpart approach, we have not been able to observe a self-replicating phase. In such phase which appears above a critical radius of an expanding ring, spot-like localized structures grow, deform and make replicas of themselves until they occupy the entire space. Within the stochastic framework, this phase is replaced by a phenomenon where an expending ring eventually breaks into two or more slowly expanding croissants repealing each other. These structures reach the boundary of simulating box without further dividing even if the size is twice the one of Figures 7-(d).

When rings expand, they suck the regulator $V$ from the corona in front of the moving interface. The consequence of this process is that when two rings collide, they start to interact at a distance larger than the 'hardcore' radius. This is shown in Fig. 8 which reproduces snapshots of two expanding rings in two generic cases. The top panel corresponds to the situation where the two rings first deform and repeal each other. The bottom panel corresponds to an annihilation dynamics where two expanding rings fuse together in one, so that small-scale features are also reproduced in the stochastic framework. In general, however, two expanding rings tend to avoid each other, cease from having a circular shape and repeal each other.

\section{Clusters serve to digitize biochemical signals in the diffusion-uncoupled case}

The model developed so far provides a simple mechanism for nucleation of IR clusters in the absence of cytoskeleton. 
When adhesion sites mature, local production of $\mathrm{PIP}_{2}$ not only controls IR clustering but also controls actin dynamics to provide strong binding sites for IRs. Consequently, actin dynamics confiscate $\mathrm{PIP}_{2}$ molecules and limit IR recruitment. In the usual case where adhesive complexes are much larger than the nascent adhesions studied so far, a simple model consists in limiting local production of $\mathrm{PIP}_{2}$ with a finite density of ligands $\mathrm{L}$ distributed in an adhesive disk as in Fig. 9.

Ligands immobilize active IRs as

$$
\mathrm{I}_{a}+\mathrm{L} \underset{1_{-}}{\stackrel{1_{+}}{\rightleftarrows}} \mathrm{I}_{a}-\mathrm{L}
$$

with kinetic rates $1_{ \pm}$. In what follows, only the species $\mathrm{I}_{a}-\mathrm{L}$ form with $\mathrm{V}$ a complex which produces $\mathrm{U}$. When $\mathrm{L}$ is in very large excess, this intermediate step is irrelevant, since active IRs can always find available ligands. In parallel, the irreversible step (5) is now taken as

$$
\mathrm{I}_{a}-\mathrm{L}-\mathrm{V} \stackrel{1 / \tau}{\longrightarrow} \mathrm{I}-\mathrm{L}+\varnothing
$$

where $\tau$ is the dwell time of the complex $\mathrm{I}-\mathrm{L}-\mathrm{V}$ producing the activator $U$. (15) can be further generalized by adding another reaction step $\mathrm{I}_{a}-\mathrm{L}-\mathrm{V} \stackrel{1 / \tau}{\longrightarrow} \mathrm{I}_{a}+\mathrm{L}+\varnothing$ without change in the following.

To avoid spurious boundary effects where the activator is produced only at the rim of the adhesive disk, the diffusion of $\mathrm{V}$ and I must be much faster than the immobilization rate for binding $\mathrm{I}_{a}$ to $\mathrm{L}$ or $\mathrm{V}$ to $\mathrm{I}-\mathrm{L}$. This sets a maximum size of an adhesive disk controlled by the density $\mathrm{L}_{0}$ of available ligands. In this diffusion-uncoupled regime, the adhesive disk is homogeneously populated by inactive receptors. The system can therefore take advantage of freely diffusive IRs to increase locally active receptors by a burst of activator.

As shown in Fig. 9, IR clusters are clearly observable at low enough rate of production $g_{\text {prod }}$ where not all ligands are occupied. Increasing this rate drives the system to the fully occupied regime in a step wise manner, see Fig. 10. This is explained in Appendix D, where the system is shown to be analogous to a $\phi^{3}$ theory with a threshold blurred by finite size effects. Membrane diffusive components can, therefore, digitize signals by controlling the density of immobilization sites. Clearly, even in the low production rate regime, the feedback loop is enough to give small clusters of active IRs near the sites of production of $u$.

\section{IR crosstalk}

The same feedback mechanism can also be generalized to the case of a mixture of different families of IRs. A key regulator of this crosstalk is known to be the cytoskeleton binding proteins Talin which activates IRs[27, 39] in a $\mathrm{PIP}_{2}$ dependent way. Here, this crosstalk is studied by introducing two IR families, $\mathrm{I}_{1,2}$, competing for immobilization sites with the production of the same activator $\mathrm{U}$. As expected, a small molecular asymmetry between the two IRs is amplified by the feedback loop which localizes active receptors near the site of production of $\mathrm{U}$.

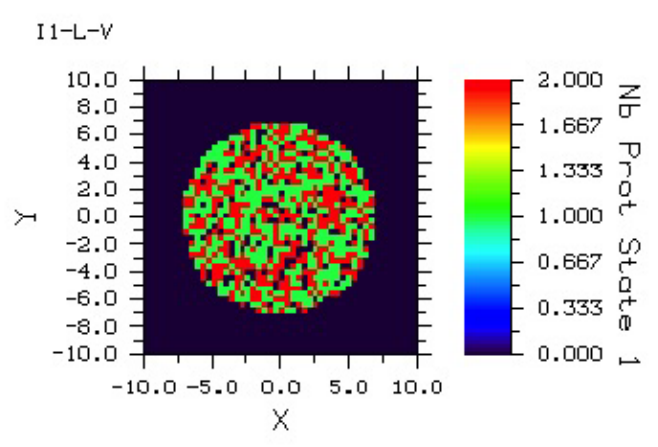

FIG. 9. Numerical simulations with an adhesive disk where receptors get immobilized by binding to ligands. The adhesive disk consists in a central disk with a finite number of ligands L per compartment, 2 in this case, and without any ligand outsidew. Only the complexes I-L can bind $\mathrm{V}$ to trigger the feedback loop. This case corresponds to the diffusion-uncoupled regime where the diffusion of $\mathrm{V}$ is much faster than its association time so that the adhesive disk is homogeneously populated with dynamic clusters of active IRs.

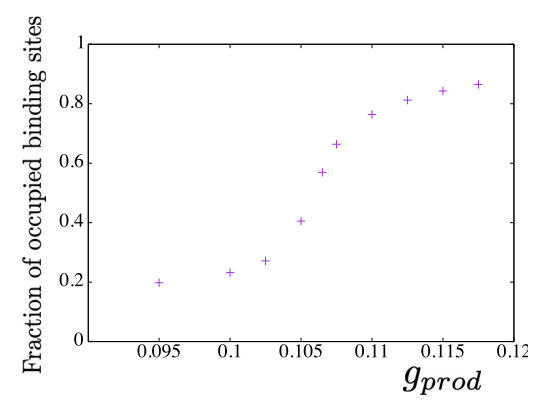

FIG. 10. Relative fraction of occupied ligand as a function of the feedback strength $1 / \varepsilon$ in the adhesive disk of the pattern of Fig. 12. The system can be tuned from a low occupation probability to a high occupation probability by a small variation of the feedback strength $\left(g_{\text {prod }}\right)$. This example corresponds to the system studied in Appendix with one ligand molecule per compartment.

A simple way to introduce this asymmetry is to take $\beta_{2}>\beta_{1}$ for the off-rates $\omega_{-}(\mathrm{U})$ with equal affinities in (14) and (15). In this case, the $I_{2}$ receptors spend more time in the active state and thus experience the slowest diffusive dynamics when they bind and unbind from the ligand. Because of the feedback, the relative concentration of active $\mathrm{I}_{2}$ receptors is considerably increased in comparison to $\mathrm{I}_{1}$ 's, and the probability for a ligand to bind an active $I_{2}$ is the highest. Ligands are thus dominantly bound to receptors of type 2 .

This is the case in Fig. 11, where the two receptors have the same unperturbed densities to simulate the same expression level. Different scenarios can be considered depending on the effective off-rate $\omega_{-}(\mathrm{U})$ of the receptors. First, in the unmixed case and in the sufficiently large off-rate limit, $\omega_{-}(\mathrm{U}) / \omega_{+}(\mathrm{U})<1$ for the $\mathrm{I}_{1}$ receptors, the fraction of occupied ligands approaches one while the fraction of unbound active IR is negligible. When $\mathrm{I}_{1}$ receptors are now mixed with $\mathrm{I}_{2}$ receptors with $\beta_{2}=2 \beta_{1}$, the $\mathrm{I}_{2}$ receptors switch off the $\mathrm{I}_{1}$ 's by binding to the L's, see Fig. 11. As a result, mixing the two 

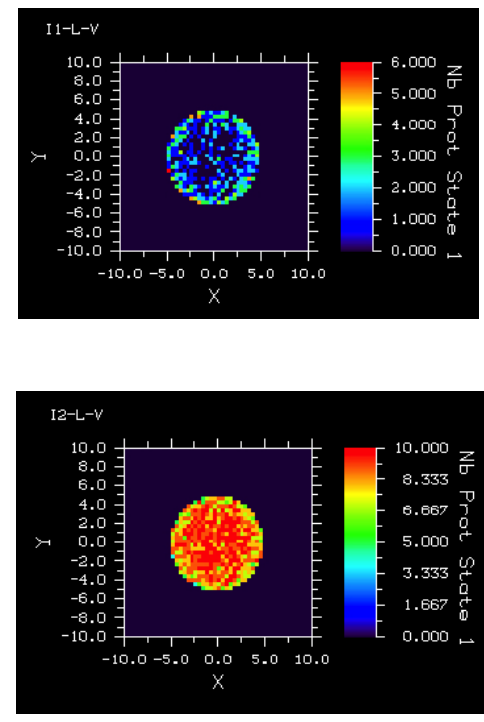

FIG. 11. A trans-regulation mechanism is a mechanism by which one receptor perturbs the binding of another receptors to a ligand. In this in example, two IRs, $\mathrm{I}_{1,2}$, compete for the same ligand $L$. The two receptors have the same parameters but differ in their effective off-rates $\omega_{-}(u)$. Due to the feedback loop which increases the concentration of the receptor with the slowest dynamics, $\mathrm{I}_{2}$, the binding of $\mathrm{I}_{1}$ to $\mathrm{L}$ is down-regulated by $\mathrm{I}_{2}$. 10 ligands per compartment.

IR families drives the $I_{1}$ 's to the inactive state so that $I_{2}$ receptors down-regulate the signaling of $\mathrm{I}_{1}$ which are now all inactive. In the other limit of not too large off-rate, the scenario is similar. The increase of $I_{2}$ due to the self-production of the activator still increases the density of the active receptors in the active state, so that the fraction of $I_{1}$ receptors bound to ligands is again negligible. However, because of the effective off-rate $\omega_{-}(\mathrm{U})$ of $\mathrm{I}_{1}$, the fraction of active $\mathrm{I}_{1}$ receptor is no more negligible so that they can bind other sites than L. In this case again, $I_{2}$ receptors regulate the binding of $I_{1}$ receptors to the ligands.

For simplicity, IR crosstalk has been analyzed in the simple scheme where the dynamics of the two receptors differ. Other mechanisms as kinase specificity or different binding times may also contribute to this crosstalk. For example, Fig. 12 demonstrates the same exclusion effect for two IR differing in their dwell times $\tau_{1,2}$ and when the kinases $V$ and $S$ are specific to the receptors. In general, the model predicts a transregulation mechanism which amplifies the concentration of IRs with the highest residence time near immobilization sites and which are therefore the most capable ones to transmit a mechanical force. Since actin polymerization and mechanical linkage are $\mathrm{PIP}_{2}$ dependent and occur in concert, favoring the IRs with the highest residence time as predicted by the model gives a recruitment mechanism for the reinforcement of force transmission to the extracellular matrix. (a)

(b)

FIG. 12. Case where ligands are patterned inside the central adhesion disk, so that IRs can only bind ligands in this adhesive disk. The total number of ligands per compartment is 10 so that the central zone of disk is exclusively populated by type $I_{2}$ receptors with the longest dwell time $\tau_{2}>\tau_{1}(\mathrm{~L}=10)$ ) for otherwise symmetric IRs. $K_{-, i}=1 / \tau_{i}, i=1,2$ for both integrin. The two kinases $V$ and $S$ are specific to $I_{1,2}$, respectively.

\section{CONCLUSION}

This paper combines conformational signaling with environment sensing to derive a model for IR clustering. Here this combination is implemented with a lifetime of a signaling receptor in its active state which depends on its membrane environment. The present approach departs from traditional Turing patterns usually described by classes of model with very different diffusivities and where the form of a polynomial non-linearity determines the selection and the stabilization of patterns[40-42]. Here, these non-linearities at the origin of IRs recruitment are fixed by a chemical potential for IR activation. Naturally, this chemical potential is modulated by the concentration of a key membrane phospholipid known to serve as a docking platform for numerous adhesive regulators. A point like excitation with a threshold gives nonequilibrium excited patterns with size corresponding to the diffusion length of this key membrane component. Clusters of receptors grow by lateral accretion and limiting factors resulting from the stress accumulation of IRs intercalating into rigid structures are neglected. This mechanism is validated in the stochastic regime and illustrates the role of robust parameters such as the size of the adhesive pattern and the ratio of the activator-regulator diffusion lengths.

To connect with cellular signaling, it may be helpful to recall that $\mathrm{PIP}_{2}$ is easily transformed by many enzymatic activities. Its diffusion length is expected to be at most a few hundred of nanometers. Because this phopholipid controls many 
cellular processes including actin polymerization[17] and integrin activation, its local production at adhesive sites is well documented[15, 43]. In particular, $\mathrm{PIP}_{2}$ is produced at adhesive complexes by the PIKI $\gamma$ kinase under the control of the kinase $\operatorname{Src}[18,20]$. Src, additionally, is inactive in the cytosol and is recruited to the membrane where it diffuses to adhesive sites where it becomes active. For simplicity, the model presented in this paper lumps together these two kinases in one single module $\mathrm{V}$ and outlines these generic recruitment rules. $\mathrm{PIP}_{2}$ can be considered as a fine regulator in the coordination of integrin since the specific ablation of PIPkI $\gamma$ at adhesive impairs only temporally integrin activation[44] but has strong effects on integrin-cytoskeleton linkages and abolishes adhesion reinforcement. This is consistent with the present model where sites of $\mathrm{PIP}_{2}$ production and sites of immobilization are spatially coordinated. Although other pathways working in synergy with $\mathrm{PIP}_{2}$ signaling $[45,46]$ are likely to occur, the feedback loop model of this paper predicts that active integrins should be coordinated and form nano-clusters as suggested in [23].

This work establishes also a formal casual relation between the dynamics of the IRs and their signaling properties, since dynamic receptors with relatively fast apparent diffusive motion can only form short-lived immobilized complexes. This principle could explain the different collective behaviors and patterning in adhesive complexes of two different IRs having slightly different binding properties. In particular, $\beta_{1}$ integrins, with slower dynamics in focal adhesive clusters than $\beta_{3}$ integrins, are spatially segregated from $\beta_{3}$ integrins in adhesive complexes[22] and podosome structures [24]. Integrin segregation in maturing adhesive complexes[47] and competition for binding talin has been invoked before as a transdominant inhibition between two integrin receptors[39]. This work links this phenomenon to $\mathrm{PIP}_{2}$ local production and gives a differential recruitment mechanism for IRs which supports models for mechanical reinforcement as in [29].

As a final point, this paper together with Ref. [26], predicts that the type of localized excited adhesive structures strongly depends on the ratio of the two diffusion lengths for the activator and regulator. One way to change this ratio and to change IR patterning behavior is to engineer synthetic molecules which are directly recruited to adhesive sites. This approach has recently been taken in Ref. [48] where the use of photo-controlable SRC kinase demonstrates that IR clusters forming adhesive complexes reorganize into podosomes under the condition of direct SRC recruitment.

\section{ACKNOWLEDGMENTS}

I thank A. Dupont and the Dysad group at the Institute for Advanced Biosciences for helpful discussions. In particular, I thank O. Destaing for his critical comments and his continuous inputs and encouragement.

\section{Appendix A: Spike solutions}

Turing patterns are generically studied in reaction diffusion systems where species have very different diffusion constants and ratio of 100 are commonly reported in stochastic studies [36]. When the diffusion length of the inhibitor is much larger than the diffusion length of the activator, an asymptotic analysis for a simpler model is tractable in one dimension[49, 50] and this analysis can only be partially extended to two-dimensional problems[51, 52] for ring solutions. The method outlines below provides a simple analytical approach for the present 2D-model valid when the diffusion lengths are comparable in magnitude and it demonstrates how this model differs qualitatively from previous ones.

Assuming symmetry of revolution, it is convenient to rewrite equations (1) as

$$
\begin{array}{r}
d_{u} \frac{1}{r} \frac{d}{d r}\left[r \frac{d u}{d r}\right]-\frac{b}{\varepsilon} u=-\frac{1}{\varepsilon} v(r) n_{a}(u) \\
d_{v} \frac{1}{r} \frac{d}{d r}\left[r \frac{d v}{d r}\right]+\tilde{h}\left(v_{h}-v\right)=v \tilde{n}_{a}(u)
\end{array}
$$

where $\tilde{h}$ and $\tilde{n}_{a}(u)$ are defined as $\tilde{h}=h+n_{a}\left(u_{h}\right)$ and $\tilde{n}_{a}(u)=$ $n_{a}(u)-n_{a}\left(u_{h}\right)$. Using this notation, the diffusion length $l_{v}=\sqrt{d_{v} / \tilde{h}}$ appears naturally, because $\tilde{n}_{a}(u)$ behaves as a Dirac delta-function. We use fixed boundary conditions with $(u, v)=\left(u_{h}, v_{h}\right)$ so that $\tilde{n}_{a}(u)$ tends to zero as $r$ goes to infinity with a spike centered at $r=0$. In (A1), we assume also the IRs have converged to their asymptotic value, so that $n_{a}(u)=n_{0} \omega_{+}^{0} / \omega_{-}^{0} e^{\beta u}, \beta=1$.

These equations can be formally solved using the Green's function technique. Recall that if $w_{0}(z), w_{1}(z)$ are two solutions of the homogeneous problem with boundary conditions $w_{0}^{\prime}(z=0)=w_{1}^{\prime}(z=\infty)=0$, then the Green's function with appropriate boundary conditions is

$$
\begin{aligned}
& G_{x}(z)=\frac{1}{x^{\mathscr{W}}(x)} w_{1}(x) w_{0}(z) \text { for } x \leq z \\
& G_{x}(z)=\frac{1}{x^{2} \mathscr{W}(x)} w_{0}(x) w_{1}(z) \text { for } x \geq z
\end{aligned}
$$

where $\mathscr{W}(x)$ is the wronskian.

For $d=2, w_{0,1}(r)$ are Bessel's functions. Then $u(r)$ solves the integral equation

$$
\begin{array}{r}
u(r)=\frac{1}{d_{u} \varepsilon}\left[K_{0}\left(r / l_{u}\right) \int_{0}^{r} z d z I_{0}\left(z / l_{u}\right) v(z) n_{a}(u)+\right. \\
\left.I_{0}\left(r / l_{u}\right) \int_{r}^{\infty} z d z K_{0}\left(z / l_{u}\right) v(z) n_{a}(u)\right]
\end{array}
$$

where $I_{0}, K_{0}$ are Bessel functions of zero order. From (A1), $v(r)$ satisfies a similar equation. Specializing to $r=0$, we have for the values of $u$ and $v$ at the maximum of the spike

$$
\begin{aligned}
& u_{s}=\frac{1}{d_{u} \varepsilon} \int_{0}^{\infty} r d r K_{0}\left(r / l_{u}\right) v(r) \tilde{n}_{a}(u)+\frac{v_{s}}{v_{h}} u_{h} \\
& v_{h}-v_{s}=\frac{1}{d_{v}} \int_{0}^{\infty} z d z K_{0}\left(z / l_{v}\right) v(r) \tilde{n}_{a}(u)
\end{aligned}
$$




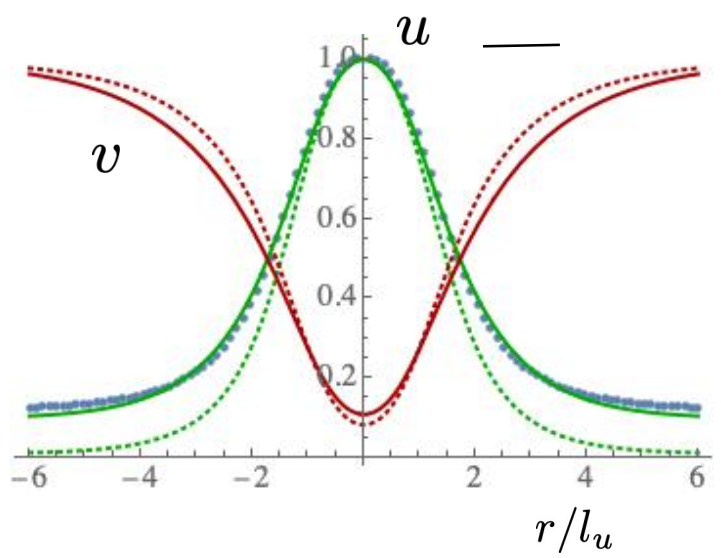

FIG. 13. Comparaison between the analytical and numerical solutions for the spike solution along the boundary line of Fig. 4. The activator $u$ and the the regulator have equal diffusion constants, $d_{u}=d_{v}=1$, with $l_{v} / l_{u}=1.68$ as indicated by the bar. The continuous lines for $u(r)$ and $v(r)$ correspond to the numerical solutions of (1). The dotted lines for $u(r)$ and $v(r)$ are the analytical solutions corresponding to $\left(u_{s}, v_{s}\right)$ solutions of (A7). Points on the continuous $u$-curve correspond to the analytical solution when (A5) is selfconsistently calculated using $v(r)$ from the analytical solution. For convenience, $u$-curves have been normalized by their maximum values which almost coincides $\left(u_{\max }=5.72\right.$ for the direct integration and $u_{\max }=5.92$ for system (A7)).

where

$$
\int_{0}^{\infty} r d r K_{0}\left(r / l_{u}\right) v(r) \approx v_{s} l_{u}^{2}
$$

since $\tilde{n}_{a}(u)=n_{a}(u)+n_{a}\left(u_{h}\right)$ in the first equation with $n_{a}\left(u_{h}\right) \ll 1$. This is enough to calculate $\left(u_{s}, v_{s}\right)$. For plotting $u(r)$ as in Fig 13, $u(r)$ must tend to $u_{h}$ as $r \gg l_{u}$. When $u_{h}$ is negligible, this does not pose problem, but to compare with numerical calculations at small but non-zero values of the activator in the quiescent state, one has to self-consistently evaluate this integral using $v(r)$ from the analytical solution for $u$ to match $u_{h}$ as $r$ goes to infinity. As expected from Fig. $13, v_{h}-v_{s}$ is well approximated by the 2D-Green's function $\propto-K_{0}\left(r / l_{v}\right)$.

Since $\tilde{n}_{a}(u)$ is strongly peaked at $r=0$ and tends to zero at infinity, all integrals in (A4) can be evaluated using the saddle-point approximation. The second derivatives of $u$ and $v$ are known from the equations of motions. Eqs. (A4) are, therefore, equations for $\left(u_{s}, v_{s}\right)$, and this system completely determines the solution. One finds for the second derivative:

$$
\begin{aligned}
& u^{\prime \prime}(0)=\frac{1}{2 l_{u}^{2}}\left[u_{s}-\frac{1}{b} v_{s} n_{a}\left(u_{s}\right)\right] \\
& v^{\prime \prime}(0)=\frac{1}{2 d_{v}}\left[v_{s} \tilde{n}_{a}\left(u_{s}\right)-\tilde{h}\left(v_{h}-v_{s}\right)\right]
\end{aligned}
$$

and all high order derivatives can be calculated in the same way.

Truncating this expansion to the second order gives only an approximation for the integrals but is enough to describe the bifurcation to the spike. One can now approximate $u(r)$ and $v(r)$ in (A4) and use definition (C1) of Appendix $\mathrm{C}$ to find

$$
\begin{aligned}
& u_{s}=\frac{v_{s} \tilde{n}_{a}\left(u_{s}\right)}{b} F\left(\frac{w_{s}}{4}, l_{u}^{2} / d_{v}\right)+\frac{v_{s}}{v_{h}} u_{h} \\
& v_{h}-v_{s}=\frac{v_{s} \tilde{n}_{a}\left(u_{s}\right)}{\tilde{h}} F\left(\frac{l_{v}^{2}}{l_{u}^{2}} \frac{w_{s}}{4}, \frac{l_{v}^{2}}{l_{u}^{2}} \frac{l_{u}^{2}}{d_{v}}\right)
\end{aligned}
$$

where $w_{s}=v_{s} \tilde{n}_{a}\left(u_{s}\right) / b-u_{s}$ is twice the magnitude of the curvature of $u$ at $r=0$ scaled by $l_{u}^{2}$ with definition (C3) for $F$.

To summarize, the saddle point approximation is a used as substitution method to replace the problem of finding a set of integral solutions by the problem of finding the solutions of an algebraic system. Once the values $u_{s}$ and $v_{s}$ are determined, the full solution can be obtained from (A3) using again the saddle point approximation. The main difference with the one-dimensional calculation $[49,50]$ is that the curvature of $v(r)$ in not negligible in two dimensions even in the limit $l_{v} / l_{u} \gg 1$, so that $v(r)$ cannot be considered as constant within the spike.

\section{Appendix B: Results for the spike solution}

In general, Eqs. (A7) can only be numerically solved. To proceed, the contour plots for the $\left(u_{s}, v_{s}\right)$ solutions of these equations are plotted and the intersection points determined. Fig. 14 demonstrates the generic behavior of these solutions as the ratio $d_{v} /\left(d_{u} \varepsilon\right)$ is varied. When this ratio is sufficiently large, the lateral diffusion of $v$ is large enough to compensate for the loss of $v$ within the spike. The system possesses two solutions at low and large values of the activator $u_{s}$ in the spike. The small $u_{s}$ solution approximates reasonably the numerical simulation, see Figs. 13 and 15, while the large $u_{s}$ solution of (A7) is almost singular within the spike. This observation is consistent with a saddle-node bifurcation where a stable and unstable solutions merge together along the boundary line and cease to exist for lower ratio of $d_{v} /\left(d_{u} \varepsilon\right)$ when lateral diffusion is not strong enough. One interesting consequence of the saddle approximation is that the scaling of (A7) gives the geometry for the boundary line in Fig. 4 between the homogeneous state and the spike solution. Since the parameters $\varepsilon$ and $d_{v}$ enter only through the combination $\varepsilon / d_{v}$, the existence of solutions is only conditional to this ratio when both parameters are varied. As a result, the bifurcation between the homogeneous and the spike phases occurs along a line in the $\left(\varepsilon, d_{v} / d_{u}\right)$ plane for the phase diagram of Fig. 4.

To derive approximate asymptotic results, it is useful to approximate (A7) as

$$
\begin{aligned}
u_{s} & =\left(u_{s}+w_{s}\right) F\left(w_{s} / 4, l_{u}^{2} / d_{v}\right) \\
\frac{\tilde{h} v_{h}}{b} & =\left(u_{s}+w_{s}\right) F\left(l_{v}^{2} w_{s} /\left(4 l_{u}^{2}\right), l_{v}^{2} / d_{v}\right)
\end{aligned}
$$

since $v_{s} \ll v_{h}$ and to consider the asymptotic expansions derived in Appendix C. In practice, (B1) is a very good approximation to (A7). 


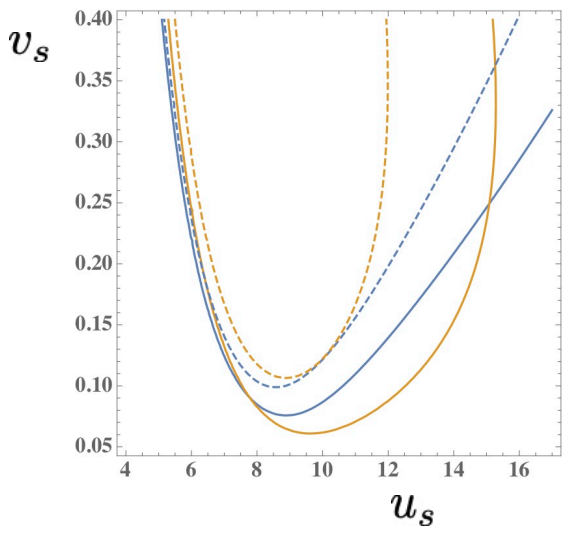

FIG. 14. Contour plot of the two self-consistent equations (A7) in the $\left(u_{s}, v_{s}\right)$ plane for different values of $d_{v} / d_{u}$. For sufficiently large values of $d_{v}$ (continuous lines) the problem gives two intersection points at low and large values of $u_{s}$. The small $u$ solution corresponds to case (a) of Fig. 15, while the large $u$ solution has much stronger variations (not shown). Decreasing $d_{v}(\times 0.8$ for this case), the two solutions merge at a single point and ceases to exist below a threshold for $d_{v} / d_{u} \varepsilon$ (dashed lines) corresponding to the homogeneous-spike boundary line at the right of the phase digram of Fig. 4. Compared with the direct integration method of Fig. 4-(a), this approach gives $\left(u_{s}, v_{s}\right) \approx(7.4,0.1)$ instead of $(7.85,0.16)$ from the direct numerical integration of the system.

Taking the ratio of the last two equations gives for $u_{s}$

$$
u_{s}=\frac{\tilde{h} v_{h}}{b} \frac{F\left(w_{s} / 4, l_{u}^{2} / d_{v}\right)}{F\left(l_{v}^{2} w_{s} /\left(4 l_{u}^{2}\right), l_{v}^{2} / d_{v}\right)}
$$

Ignoring logarithmic corrections of order $\ln \left(l_{v}^{2} / l_{u}^{2}\right)$, see Appendix $\mathrm{C}$, one obtains a characteristic scale for the activator within the spike

$$
u_{s} \approx \frac{l_{v}^{2}}{l_{u}^{2}} \frac{\tilde{h} v_{h}}{b}=\frac{d_{v}}{d_{u}} \frac{v_{h}}{\varepsilon}
$$

In practice, this expression overestimates the numerical result by a factor of order two, so that comparaison with the numerical calculation is only possible by numerically solving (B1). This asymptotic expression gives, however, a lower bound for $d_{v} /\left(d_{u} \varepsilon\right)$ under which the spike solution ceases to exist. To derive it, it suffices to require that $u_{h}$ must be larger than some threshold set by the natural rates of the IRs. One finds

$$
\frac{d_{v}}{d_{u}}>\frac{\varepsilon}{v_{h}} \ln \left[\frac{\omega_{-}^{0}}{\omega_{+}^{0}}\right]
$$

which overestimates the apparent slope of Fig. 4 by a factor 2 (where $v_{h} \approx 6.4$ ).

In conclusion, this method allows to identify characteristic orders of magnitude for the activator in the spike and for the boundary line between the homogeneous and the spike phase of Fig. 4.
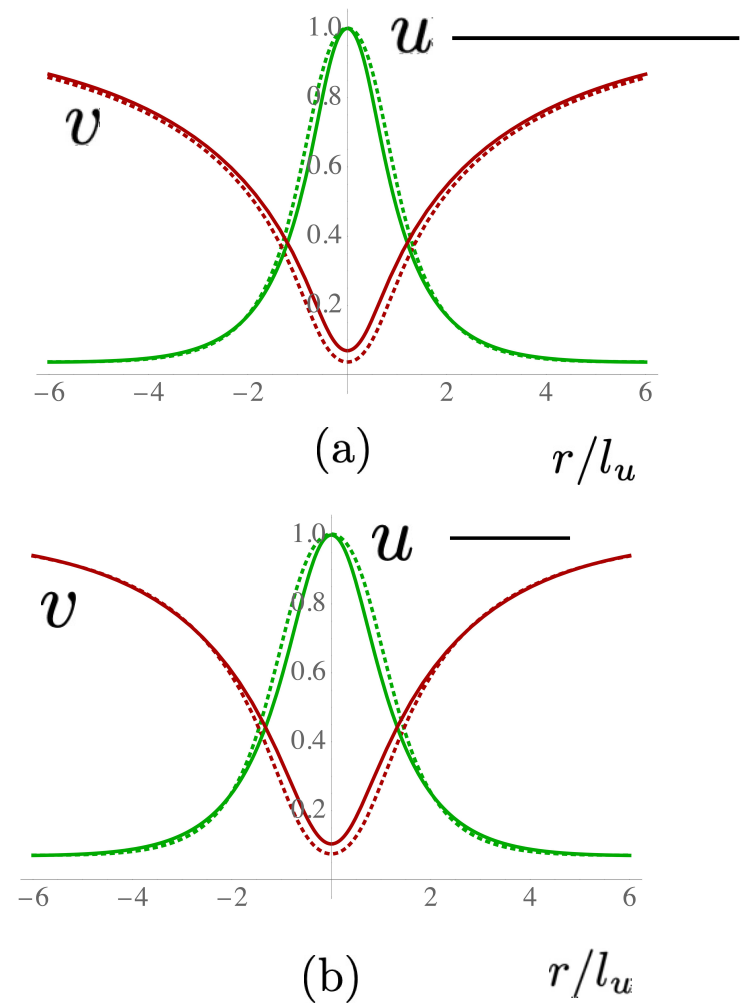

FIG. 15. Examples of spike solutions with symmetry of revolution for decreasing ratios of the diffusion lengths $l_{v} / l_{u}$ indicated by the thick bar. From the top to the down, (a) $l_{v} / l_{u}=5.26$, (b) $l_{v} / l_{u}=$ 2.80. The continuous lines, with a maximum at the center of the spike, correspond to the activator $u$. The continuous lines, with a minimum, are plots of the regulator $v$. The dotted lines correspond to the saddle solutions for the same parameters. Cases (a) and (b) are for parameters on the boundary lines with the homogeneous phase, see Fig. 4. For graphical reasons, the plots of the activator $u$ have been normalized by the same value $u(0)$, the saddle approximation (A7) giving the same result as the numerical solution within a few percent ( $h=0.02, b=0.1,1 / \varepsilon=4.65$ for the top curve and $h=0.05, b=0.1$ and $1 / \varepsilon=2.6$ for the bottom one).

\section{Appendix C: An integral}

For what follows, it will be useful to consider the following integral, see [53], formula (6.63)

$$
\begin{aligned}
F_{n}(\alpha) & =\int_{0}^{\infty} x^{n} d x K_{0}(x) e^{-\alpha x^{2}} \\
& =\frac{1}{2 \alpha^{n / 2}} e^{\frac{1}{8 \alpha}}\left[\Gamma\left(\frac{n+1}{2}\right)\right]^{2} W_{-\frac{n}{2}, 0}\left(\frac{1}{4 \alpha}\right)
\end{aligned}
$$

When $\alpha$ is large, the following expansions hold

$$
\begin{aligned}
& F_{1}(\alpha) \sim(\ln (4 \alpha)-\gamma) /(4 \alpha)+\ldots \\
& F_{3}(\alpha) \sim(\ln (4 \alpha)-\gamma-1) /\left(4 \alpha^{2}\right)+\ldots
\end{aligned}
$$

where $\gamma=0.577276 \ldots$ is the Euler constant. 
As in (A7), one defines

$$
F\left(w, l_{u}^{2} / d_{v}\right)=F_{1}(w)+\frac{l_{u}^{2}}{4 d_{v} v_{s}}\left(v_{s} \tilde{n}_{a}\left(u_{s}\right)-\tilde{h}\left(v_{h}-v_{s}\right)\right) F_{3}(w)
$$

From the numerical point of view, $F_{1}(\alpha) /\left(\alpha F_{3}(\alpha)\right)$ approaches 1 only in the limit where $\ln (\alpha)$ is sufficiently large, so that strong logarithmic corrections to (B3) are expected.

\section{Appendix D: Current conservation and $\phi^{3}$ theory}

This Appendix demonstrates the equivalence of the model with a $\phi^{3}$ theory in the asymptotic limit where the activation of the feedback loop is simplified by introducing ligands for receptor immobilization. In the limit we consider, the limiting factor for the production of $u$ is set by the number of ligands, so that we disregard the regulator $\mathrm{V}$ assuming that all $\mathrm{I}-\mathrm{L}$ complexes are bound to $\mathrm{V}$. We introduce the molecular scheme

$$
\mathrm{I}_{a}+\mathrm{L} \underset{k_{-}}{\stackrel{k_{+}}{\rightleftharpoons}} \mathrm{I}-\mathrm{L}
$$

where an activated receptor binds an immobilized ligand to form a complex I-L. Conservation of ligands in each compartment implies the sum of complexed and free ligands must be constant, $\mathrm{L}+\mathrm{I}-\mathrm{L}=\mathrm{L}_{0}$. As in the text, we assume that ligands are patterned inside an adhesion disk of size $R$ and that diffusion is sufficiently fast so that the concentration of bound receptors is homogeneously distributed inside the adhesive disk. We write $I_{a, \text { out }}$ or $I_{a \text {,in }}$ for the concentration of activated receptors outside and inside the adhesion disk. As before, the $I_{i, \ldots}$ 's correspond to inactive receptors.

Equilibrium between the active and inactive states of the receptors implies

$$
\omega_{+}(u) I_{i, i n}-\omega_{-}(u) I_{a, i n}=0
$$

where $u$ is the concentration of activator inside the disk. A similar relation holds outside the disk and

$$
I_{a, \text { out }}=\frac{\omega_{+}(0)}{\omega_{-}(0)} I_{i, \text { out }}
$$

with

$$
\frac{\omega_{+}(0)}{\omega_{-}(0)} \ll 1
$$

Finally, conservation of ligands implies that the number of complexe I-L scales as

$$
\mathrm{I}-\mathrm{L}=\frac{k_{+} I_{a, i n}}{k_{+} I_{a, i n}+k_{-}} \mathrm{L}_{0}
$$

so that it suffices to know $I_{a, i n}$ to know $\mathrm{I}-\mathrm{L}$.

To proceed, we note that current conservation for receptors at the boundary of the adhesive zone implies

$$
D_{i} I_{i, \text { out }}+D_{a} I_{a, \text { out }}=D_{i} I_{i, \text { in }}+D_{a} I_{a, \text { in }}
$$

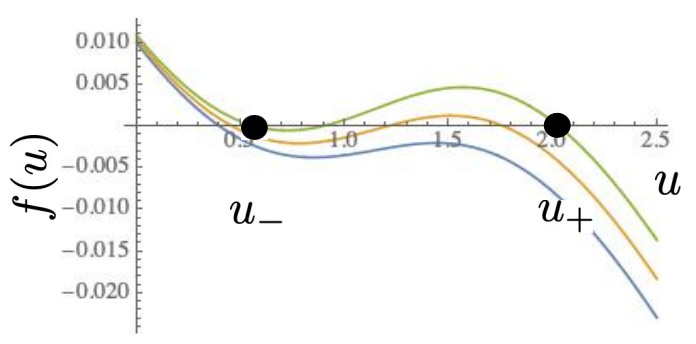

FIG. 16. Plot of the equivalent $\phi^{3}$ functional $f(u)$ for $g_{\text {prod }}=$ $0.11,0.115,0.12$, where $a$ is the rate of production of the activator as defined in Eq. D11. For comparaison with the stochastic simulations, the parameters are the same as the ones in Fig. 10. According to the Maxwell's condition, bifurcation takes place between the lowest $u_{-}$and highest $u_{+}$root of $f(u)$ when the area under the curve is zero, see (D12). Note that the continuous limit gives a larger value for the feedback strength, i.e. $\approx 0.12$, than its stochastic counterpart, i.e. $\approx 0.1075$.

where the $D_{i, a}$ 's are diffusion constants for the active or inactive forms of receptors. We will take the limit $D_{a} \ll D_{i}$ as done in text. Outside the disk, the number of activated receptor is small so that $I_{a, \text { out }}=0$. We conclude

$$
I_{i, \text { in }}=I_{i, \text { out }}\left[1+\frac{D_{a}}{D_{i}} \frac{\omega_{+}(0)}{\omega_{-}(0)} e^{\beta u}\right]^{-1}
$$

with

$$
I_{a, i n}=\frac{\omega_{+}(0)}{\omega_{-}(0)} e^{\beta u} I_{i, i n}
$$

so that we can use (D5) to find I-L.

To simplify this expression, we assume the activated receptors have small diffusion constants, $D_{i} / D_{a} \ll 1$. In that case,

$$
I-L=L_{0}\left[1+\frac{k_{-}}{k_{+} I_{a, i n}}\right]^{-1}=L_{0}\left[1+\frac{k_{-}}{k_{+}} \frac{\omega_{-}(0)}{\omega_{+}(0) I_{i, i n}} e^{-\beta u}\right]^{-1}
$$

In the fast diffusive regime, $I_{i, \text { in }}=I_{i, \text { out }}$ is constant independent of $u$. Because $I-L$ is a function of $u$, we write $\mathrm{I}-\mathrm{L}=\mathrm{I}-\mathrm{L}(u)$ in (D9).

Assuming furthermore that the rate of production of $u$ is proportional to I-L with a proportionality constant $g_{\text {prod }}$, the equation of $u$ takes the form

$$
\frac{\partial u}{\partial t}=d_{u} \Delta u+f(u)
$$

with

$$
f(u)=-b_{\text {deg }} u+g_{\text {prod }} \mathrm{I}-\mathrm{L}(u)
$$

where the irreversible degradation rate constant $b_{\text {deg }}$ plays the same role as $b / \varepsilon$ in the text. Plots of $f(u)$ are graphed in 
Fig. 16 and demonstrate that the rate of activation of $u$ has the form of a single reaction diffusion with the property of bistability, since it admits two steady states given by the zeros $u_{ \pm}$of $f(u)$. Then, it is known that the stability criterion for switching from one solution to the other one is given by the Maxwell condition[34]

$$
\int_{u_{-}}^{u_{+}} f(u) d u=0
$$

which can be meet by varying the rate of production $g_{\text {prod }}$. Comparison with the stochastic calculation of Fig. 10, see Fig. 16 , demonstrates that the slight difference for the threshold value of $a$ can be easily explained using finite sizes scaling arguments as in critical phenomena.

Finally, it is interesting to investigate the large off-rate limit

$$
\frac{k_{-}}{k_{+} I_{i, i n}} \gg 1
$$

with a large number of ligands such that the ratio $L_{0} / k_{-}$stays finite. Then, by (D5) $I-L$ is finite and we neglect the changes in the concentration of ligands.

This means that the critical ratio for the effective functional in (D9)

$$
\frac{k_{-}}{k_{+} I_{i, i n}} \frac{\omega_{-}(0)}{\omega_{+}(0)} e^{-\beta u} \gg 1
$$

is much larger than 1. In this limit, the equivalent Landau functional has an equivalent functional form of the one studied in the first part of this paper, or

$$
-b u+a I_{i, i n} \frac{k_{+}}{k_{-}} \frac{\omega_{+}(0)}{\omega_{-}(0)} e^{\beta u}
$$

[1] A. M. Turing, The chemical basis of morphogenesis, Philos Transact B, biological sciences 237, 37 (1952).

[2] M. C. Cross and P. C. Hohenberg, Pattern formation outside of equilibrium, Rev. Mod. Phys. 65, 851 (1993).

[3] A. J. Koch and H. Meinhardt, Biological pattern formation: from basic mechanisms to complex structures, Rev. Mod. Phys. 66, 1481 (1994).

[4] S. Kondo and T. Miura, Reaction-diffusion model as a framework for understanding biological pattern formation, Science 329, 1616 (2010).

[5] J. Halatek, F. Brauns, and E. Frey, Self-organization principles of intracellular pattern formation, Philos Trans R Soc Lond B Biol Sci 373, 10.1098/rstb.2017.0107 (2018).

[6] C. Albigès-Rizo, O. Destaing, B. Fourcade, E. Planus, and M. R. Block, Actin machinery and mechanosensitivity in invadopodia, podosomes and focal adhesions, J Cell Sci 122, 3037 (2009).

[7] R. O. Hynes, Integrins: bidirectional, allosteric signaling machines., Cell 110, 673 (2002).

[8] R. Changede, X. Xu, F. Margadant, and M. P. Sheetz, Nascent integrin adhesions form on all matrix rigidities after integrin activation, Dev Cell 35, 614 (2015).

[9] M. J. Paszek, D. Boettiger, V. M. Weaver, and D. A. Hammer, Integrin clustering is driven by mechanical resistance from the glycocalyx and the substrate, PLoS Comput Biol 5, e1000604 (2009).

[10] M. J. Paszek, C. C. DuFort, O. Rossier, R. Bainer, J. K. Mouw, K. Godula, J. E. Hudak, J. N. Lakins, A. C. Wijekoon, L. Cassereau, M. G. Rubashkin, M. J. Magbanua, K. S. Thorn, M. W. Davidson, H. S. Rugo, J. W. Park, D. A. Hammer, G. Giannone, C. R. Bertozzi, and V. M. Weaver, The cancer glycocalyx mechanically primes integrin-mediated growth and survival, Nature 511, 319 (2014).

[11] E. Roob, 3rd, N. Trendel, P. Rein Ten Wolde, and A. Mugler, Cooperative clustering digitizes biochemical signaling and enhances its fidelity, Biophys J 110, 1661 (2016).

[12] W. R. Holmes, B. Lin, A. Levchenko, and L. Edelstein-Keshet, Modelling cell polarization driven by synthetic spatially graded rac activation, PLoS Comput Biol 8, e1002366 (2012).

[13] J. Das, M. Kardar, and A. K. Chakraborty, Positive feedback regulation results in spatial clustering and fast spreading of ac- tive signaling molecules on a cell membrane, The Journal of Chemical Physics 130, 245102 (2009).

[14] M. Wehrens, P. R. ten Wolde, and A. Mugler, Positive feedback can lead to dynamic nanometer-scale clustering on cell membranes, J Chem Phys 141, 205102 (2014).

[15] G. Di Paolo and P. De Camilli, Phosphoinositides in cell regulation and membrane dynamics, Nature 443, 651 EP (2006).

[16] J. Saarikangas, H. Zhao, and P. Lappalainen, Regulation of the actin cytoskeleton-plasma membrane interplay by phosphoinositides, Physiol Rev 90, 259 (2010).

[17] P. A. Janmey, R. Bucki, and R. Radhakrishnan, Regulation of actin assembly by pi(4,5)p2 and other inositol phospholipids: An update on possible mechanisms, Biochem Biophys Res Commun 506, 307 (2018).

[18] G. Di Paolo, L. Pellegrini, K. Letinic, G. Cestra, R. Zoncu, S. Voronov, S. Chang, J. Guo, M. R. Wenk, and P. De Camilli, Recruitment and regulation of phosphatidylinositol phosphate kinase type 1 [gamma] by the ferm domain of talin, Nature $\mathbf{4 2 0}$, 85 (2002).

[19] V. Martel, C. Racaud-Sultan, S. Dupe, C. Marie, F. Paulhe, A. Galmiche, M. R. Block, and C. Albiges-Rizo, Conformation, localization, and integrin binding of talin depend on its interaction with phosphoinositides., J Biol Chem 276, 21217 (2001).

[20] K. Ling, R. L. Doughman, A. J. Firestone, M. W. Bunce, and R. A. Anderson, Type i gamma phosphatidylinositol phosphate kinase targets and regulates focal adhesions, Nature 420, 89 (2002).

[21] F. Saltel, E. Mortier, V. P. Hytönen, M.-C. Jacquier, P. Zimmermann, V. Vogel, W. Liu, and B. Wehrle-Haller, New pi(4,5)p2and membrane proximal integrin-binding motifs in the talin head control beta3-integrin clustering, J Cell Biol 187, 715 (2009).

[22] O. Rossier, V. Octeau, J.-B. Sibarita, C. Leduc, B. Tessier, D. Nair, V. Gatterdam, O. Destaing, C. Albigès-Rizo, R. Tampé, L. Cognet, D. Choquet, B. Lounis, and G. Giannone, Integrins $\beta_{1}$ and $\beta_{3}$ exhibit distinct dynamic nanoscale organizations inside focal adhesions, Nat Cell Biol 14, 1057 (2012).

[23] M. Spiess, P. Hernandez-Varas, A. Oddone, H. Olofsson, H. Blom, D. Waithe, J. G. Lock, M. Lakadamyali, and S. Strömblad, Active and inactive integrins seg- 
regate into distinct nanoclusters in focal adhesions, The Journal of Cell Biology 217, 1929 (2018), http://jcb.rupress.org/content/217/6/1929.full.pdf.

[24] O. Destaing, E. Planus, D. Bouvard, C. Oddou, C. Badowski, V. Bossy, A. Raducanu, B. Fourcade, C. Albiges-Rizo, and M. R. Block, $\beta_{1}$ integrin is a master regulator of invadosome organization and function, Mol Biol Cell 21, 4108 (2010).

[25] M. B. M. Meddens, E. Pandzic, J. A. Slotman, D. Guillet, B. Joosten, S. Mennens, L. M. Paardekooper, A. B. Houtsmuller, K. van den Dries, P. W. Wiseman, and A. Cambi, Actomyosin-dependent dynamic spatial patterns of cytoskeletal components drive mesoscale podosome organization, Nat Commun 7, 13127 (2016).

[26] M. R. Block, O. Destaing, C. Petropoulos, E. Planus, C. Albigès-Rizo, and $\mathrm{B}$. Fourcade, Integrin-mediated adhesion as self-sustained waves of enzymatic activation, Phys. Rev. E 92, 042704 (2015).

[27] A. M. Gonzalez, R. Bhattacharya, G. W. deHart, and J. C. R. Jones, Transdominant regulation of integrin function: mechanisms of crosstalk, Cell Signal 22, 578 (2010).

[28] K. R. Legate, S. Takahashi, N. Bonakdar, B. Fabry, D. Boettiger, R. Zent, and R. Fässler, Integrin adhesion and force coupling are independently regulated by localized ptdins(4,5)(2) synthesis, EMBO J 10.1038/emboj.2011.332 (2011).

[29] A. Elosegui-Artola, E. Bazellières, M. D. Allen, I. Andreu, R. Oria, R. Sunyer, J. J. Gomm, J. F. Marshall, J. L. Jones, $\mathrm{X}$. Trepat, and P. Roca-Cusachs, Rigidity sensing and adaptation through regulation of integrin types, Nat Mater 13, 631 (2014).

[30] A. Elosegui-Artola, R. Oria, Y. Chen, A. Kosmalska, C. PérezGonzález, N. Castro, C. Zhu, X. Trepat, and P. Roca-Cusachs, Mechanical regulation of a molecular clutch defines force transmission and transduction in response to matrix rigidity, Nat Cell Biol 10.1038/ncb3336 (2016).

[31] See Supplemental Material at http: ... for movies.

[32] J. Li and T. A. Springer, Integrin extension enables ultrasensitive regulation by cytoskeletal force, Proc Natl Acad Sci U S A 114, 4685 (2017).

[33] A. C. E. Shibata, T. K. Fujiwara, L. Chen, K. G. N. Suzuki, Y. Ishikawa, Y. L. Nemoto, Y. Miwa, Z. Kalay, R. Chadda, K. Naruse, and A. Kusumi, Archipelago architecture of the focal adhesion: membrane molecules freely enter and exit from the focal adhesion zone, Cytoskeleton (Hoboken) 69, 380 (2012).

[34] J. Murray, Mathematical Biology (Springer-Verlag, Berlin Heidelberg, 1989).

[35] R. Erban, S. J. Chapman, and P. K. Maini, A practical guide to stochastic simulations of reaction-diffusion process, arxiv preprint arxiv:0704.1908.

[36] Y. Cao and R. Erban, Stochastic Turing patterns: analysis of compartment-based approaches, Bull Math Biol 76, 3051 (2014).

[37] M. Vigelius and B. Meyer, Stochastic simulations of pattern formation in excitable media, PLoS One 7, e42508 (2012).
[38] C. B. Muratov, E. Vanden-Eijnden, and W. E., Self-induced stochastic resonance in excitable systems, Physica D: Nonlinear Phenomena 210, 227 (2005).

[39] D. A. Calderwood, V. Tai, G. Di Paolo, P. De Camilli, and M. H. Ginsberg, Competition for talin results in trans-dominant inhibition of integrin activation, J Biol Chem 279, 28889 (2004).

[40] H. Meinhardt and A. Gierer, Pattern formation by local selfactivation and lateral inhibition, Bioessays 22, 753 (2000).

[41] M. Ward, Asymptotic methods for reaction-diffusion systems: Past and present, Bull Math Biol 68, 1151 (2006).

[42] J. Allard and A. Mogilner, Traveling waves in actin dynamics and cell motility, Current Opinion in Cell Biology 25, 107 (2013).

[43] K. R. Legate and R. Fässler, Mechanisms that regulate adaptor binding to beta-integrin cytoplasmic tails, J Cell Sci 122, 187 (2009).

[44] K. R. Legate, S. Takahashi, N. Bonakdar, B. Fabry, D. Boettiger, R. Zent, and R. Fässler, Integrin adhesion and force coupling are independently regulated by localized ptdins(4,5)2synthesis, The EMBO Journal 30, 4539 (2011).

[45] J. Yang, L. Zhu, H. Zhang, J. Hirbawi, K. Fukuda, P. Dwivedi, J. Liu, T. Byzova, E. F. Plow, J. Wu, and J. Qin, Conformational activation of talin by riam triggers integrin-mediated cell adhesion, Nat Commun 5, 5880 (2014).

[46] A. R. Gingras, F. Lagarrigue, M. N. Cuevas, A. J. Valadez, M. Zorovich, W. McLaughlin, M. A. Lopez-Ramirez, N. Seban, K. Ley, W. B. Kiosses, and M. H. Ginsberg, Rap1 binding and a lipid-dependent helix in talin f1 domain promote integrin activation in tandem, J Cell Biol 10.1083/jcb.201810061 (2019).

[47] E. Zamir, M. Katz, Y. Posen, N. Erez, K. M. Yamada, B. Z. Katz, S. Lin, D. C. Lin, A. Bershadsky, Z. Kam, and B. Geiger, Dynamics and segregation of cell-matrix adhesions in cultured fibroblasts, Nat Cell Biol 2, 191 (2000).

[48] A. Kerjouan, C. Boyault, C. Oddou, E. Hiriart-Bryant, A. Grichine, A. Kraut, M. Pezet, M. Balland, E. Faurobert, I. Bonnet, Y. Coute, B. Fourcade, C. AlbigesRizo, and O. Destaing, Molecular flux control encodes distinct cytoskeletal responses by specifying SRC signaling pathway usage, bioRxiv 10.1101/648030 (2019), https://www.biorxiv.org/content/early/2019/05/25/648030.full.pdf.

[49] C. B. Muratov and V. V. Osipov, Spike autosolitons in the GrayScott model, Tech. Rep. (arxiv:patt-sol/9804001v4, 2002).

[50] C. B. Muratov and V. Osipov, Stability of the static spike autosilitons in the gray-scott model, SIAM Journal on Applied Mathematics 62, 1463 (2002).

[51] D. S. Morgan and T. J. Kaper, Axisymmetric ring solutions of the $2 \mathrm{~d}$ gray-scott model and their destabilization into spots, Physica D: Nonlinear Phenomena 192, 33 (2004).

[52] T. Kolokolnikov and J. Wei, On ring-like solutions for the grayscott model: existence, instability and self-replicating rings, European Journal of Applied Mathematics , 201 (2005).

[53] I. Gradshteyn and I. Ryzhik, Table of Integrals, Series, and Products (Academic Press, 1980). 